\title{
A Novel DCA Scheme for Resource Sharing in Cellular Environments with Heterogeneous Traffic
}

\author{
Vahid Tabataba Vakili and Arash Aziminejad \\ Department of Electrical Engineering, \\ Iran University of Science \& Technology (IUST), \\ Narmak, Tehran, 16844, IRAN \\ arash1971@yahoo.com
}

\begin{abstract}
In cellular networks it is crucial to be able to use the available radio spectrum as efficiently as possible while providing a certain level of Quality of Service (QoS) for users. Emergence of miscellaneous services has dramatically increased the complexity of this problem by creating a heterogeneous traffic environment. In this paper an efficient resource allocation scheme has been proposed for cellular networks with multimedia traffic, which combines classical resource borrowing concept with a novel inter-cell resource sharing scheme between different classes of traffic. By assuming the heterogeneous offered traffic to be a combination of audio and video traffic types, it will be shown that the proposed resource allocation scheme is capable of significantly improving audio teletraffic performance of the system without imposing additional expense upon video QoS performance.
\end{abstract}

Keywords: Resource allocation, Heterogeneous traffic, Channel borrowing assignment, Teletraffic performance

\section{Introduction}

The design of cellular networks is impressively being influenced by the continuous growth in traffic volume and the emergence of new diverse services in mobile communications. The introduction of novel applications such as data delivery and real-time multimedia in $3 \mathrm{G}$ and $4 \mathrm{G}$ systems will not only significantly increase the traffic on wireless networks, but also will create a heterogeneous traffic environments $[1,2]$. To effectively utilize the bandwidth resource, a number of bandwidth assignment schemes have been proposed in order to transmit multimedia traffic in wireless networks [3, 4].

Generally speaking, a real-time video call requires considerably larger amount of bandwidth than an audio call and in contrast to both audio and video traffic, data traffic does not have real-time requirements. Furthermore, traffic characteristics of audio and video calls (e.g. call arrival rate and call holding time distribution) are different. In practice, bandwidth resources are pre-assigned to each type of traffic and the idle capacity allocated to video or audio traffic will be temporarily used for transmitting packet switched data according to a procedure which leaves QoS performance of video and audio calls intact $[5,6]$. Keeping this preemptive priority of audio and video calls over data traffic in mind, in this research an efficient resourcesharing scheme between audio and video calls has been proposed, which is based 
upon intra-cell (for audio traffic) and inter-cell (between audio traffic and video resources) resource sharing. Since video call requests arrival rate is considerably lower comparing to audio call arrival rate and also as a video call requires higher bandwidth for transmission, the resource borrowing between audio and video calls (cross-borrowing) will be performed unilaterally, i.e. only overflowed audio calls will borrow idle video channels once by using a threshold type decision policy it has been ascertained that QoS performance of the system against video traffic will not be degraded by this resource borrowing. A criterion has been analytically derived for this purpose and numerical results obtained from extensive simulations conducted on a as real as possible cellular environment indicate that Heterogeneous Channel Borrowing Assignment with Unilateral Cross-Borrowing (HCBA-UCB) is able to reduce the audio call blocking probability considerably at the expense of only negligible increase in video call blocking. Also it will be shown that by allowing a video resource to be simultaneously borrowed by multiple audio calls on the condition that video call blocking performance requirement is not violated, teletraffic performance of HCBA$\mathrm{UCB}$ can be further enhanced.

\section{General Assignment Strategy in HCBA-UCB}

Initially all of the audio and video channels will be divided between the cells according to a pre-defined uniform Fixed Channel Assignment (FCA) reuse pattern and it is assumed that after this pre-assignment of resources each cell will possess $\mathrm{N}_{\mathrm{A}}$ audio and $\mathrm{N}_{\mathrm{v}}$ video nominal channels. Resource assignment process in HCBA-UCB follows the following algorithm:

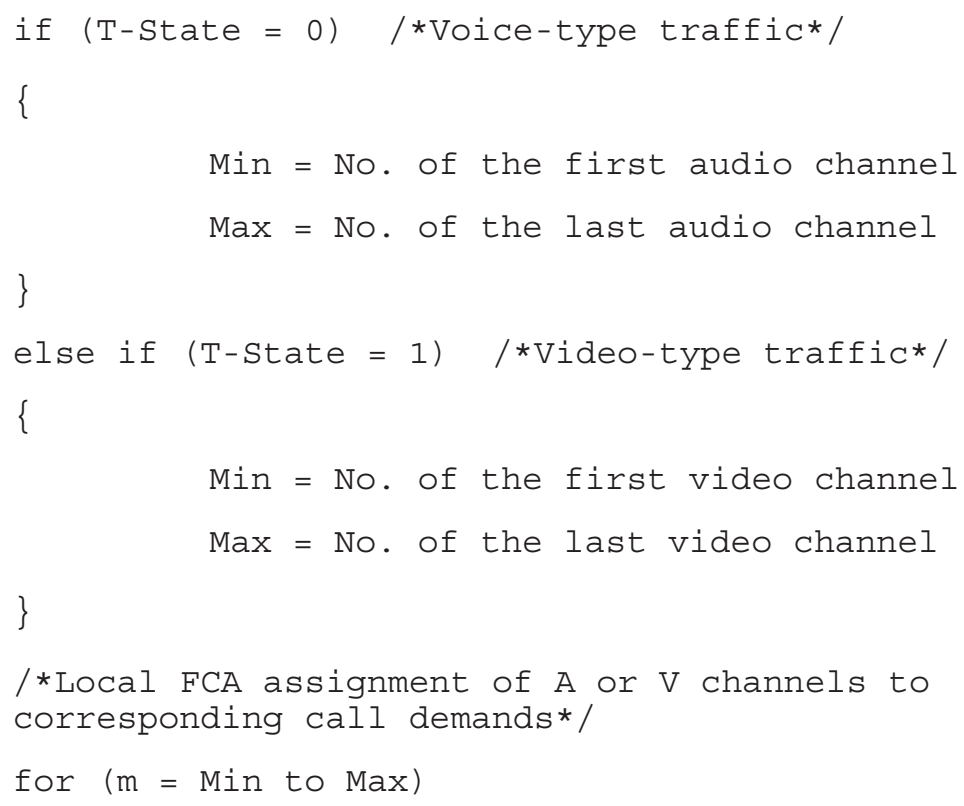




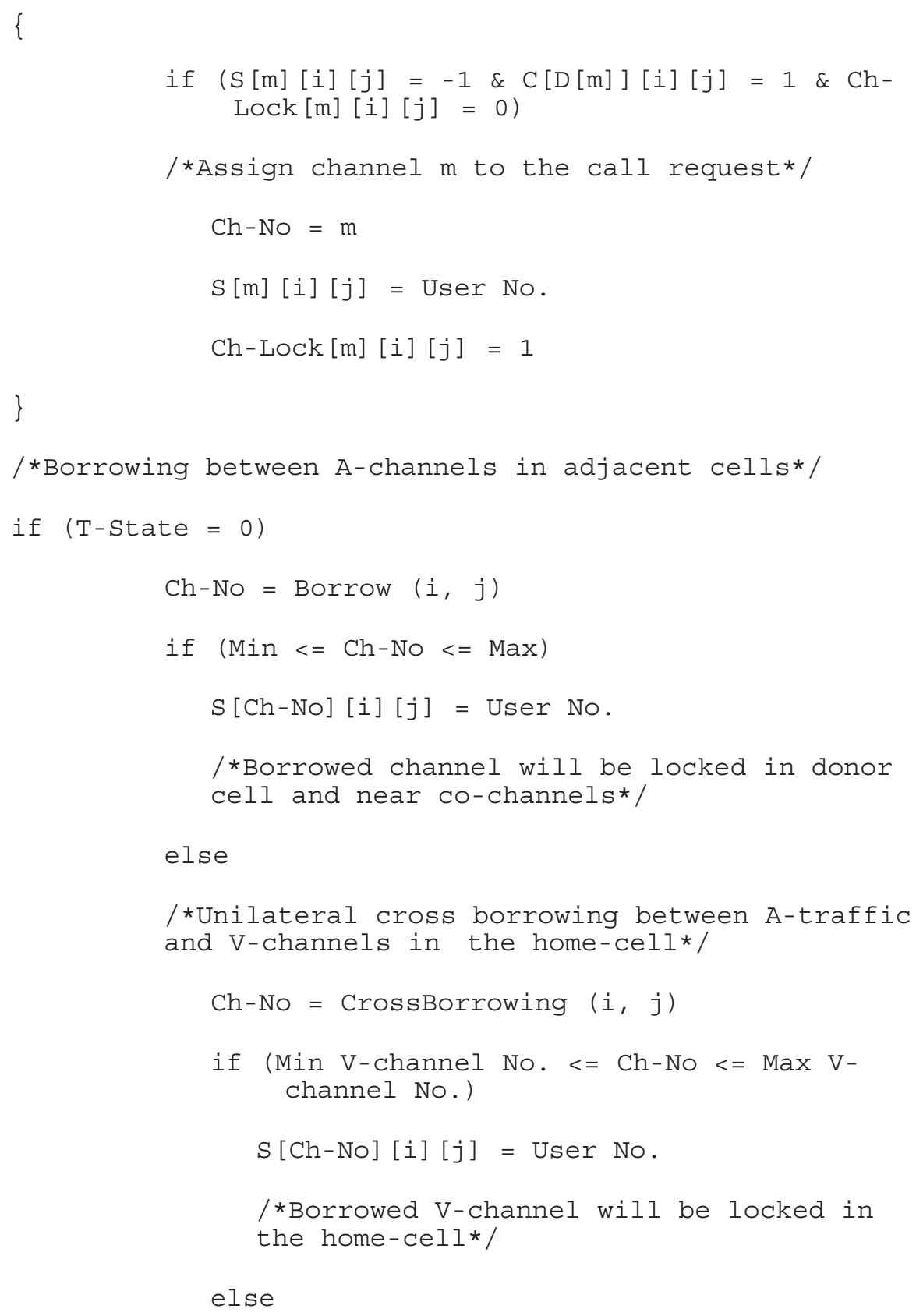

According to above-mentioned resource assignment policy, if a new video call request arrives in a cell and finds all its $\mathrm{N}_{\mathrm{v}}$ nominal video channels busy, it will be blocked. In case of a new voice call arrival, if all the $\mathrm{N}_{\mathrm{A}}$ nominal audio channels are 
busy, HCBA-UCB will try to borrow a free and unlocked audio channel from one of the six neighboring cells by activating Borrow function. The borrowing process will be accomplished based on Borrow First Available (BFA) scheme [7]. If no appropriate audio channel is found in the neighboring cells to be borrowed, HCBAUCB tries to cross-borrow one of the nominal video channels by activating CrossBorrowing function. The crucial question in permitting cross borrowing to take place is whether or not the pre-defined video call blocking probability requirement $\left(\mathrm{P}_{\mathrm{B}}\right)$ will be violated by the cross-borrowing operation. If no video channel is found to satisfy the cross-borrowing criterion in home cell, the audio call request will be blocked. To further illustrate this algorithm, A few variable names should be defined. Assume the location of cells be represented by their integer coordinates (i, j) in a twodimensional array of hexagonal cells, the m-th Compact Pattern (CP) for uniform FCA initial assignment strategy is represented by the $3 \mathrm{D}$ array $C[\mathrm{~m}][\mathrm{i}][\mathrm{j}]:[8]$

$$
C_{i j}(m)= \begin{cases}1 & \text { if cell }(i, j) \text { belongs to the } m-\text { th } C P \\ 0 & \text { otherwise }\end{cases}
$$

Array $S[k][i][j]$ denotes the occupancy state of channel $\mathrm{k}$ in cell $(\mathrm{i}, \mathrm{j})$ as:

$$
S_{i j}(k)=\left\{\begin{array}{lc}
\text { User's No. } & \text { if channel } k \text { is being used in cell }(i, j) \\
0 & \text { otherwise }
\end{array}\right.
$$

One-dimensional array $D[k]$ indicates the $\mathrm{CP}$ that has been allocated to channel $\mathrm{k}$ and it will be filled by the initial FCA assignment according to its reuse pattern [8]. Finally, Ch-Lock [k][i][j] expresses locked or unlocked state [7] of channel k in cell $(i, j)$.

$$
C h-\operatorname{Lock}_{i j}(k)= \begin{cases}1 & \text { if channel } k \text { is being locked in cell }(i, j) \\ 0 & \text { otherwise }\end{cases}
$$

\section{Unilateral Cross-Borrowing}

The unilateral cross-borrowing of video traffic resources by overflowed audio call requests in a cell can be stated in a simple way that a video channel can be crossborrowed to attend an audio call request, if sufficient number of free video channels remain after cross-borrowing so that the video call blocking in that cell does not exceeds its QoS requirement, say $\mathrm{P}_{\mathrm{B}}$. One way of approaching this problem is to use a multi-server queuing system model with queue size of zero (loss model), i.e. if no server is available at the moment that a new call originates, that call is blocked immediately. Each type of calls has different traffic parameters but all traffic sources are modeled as Poisson processes. The goal here is to find a control policy 


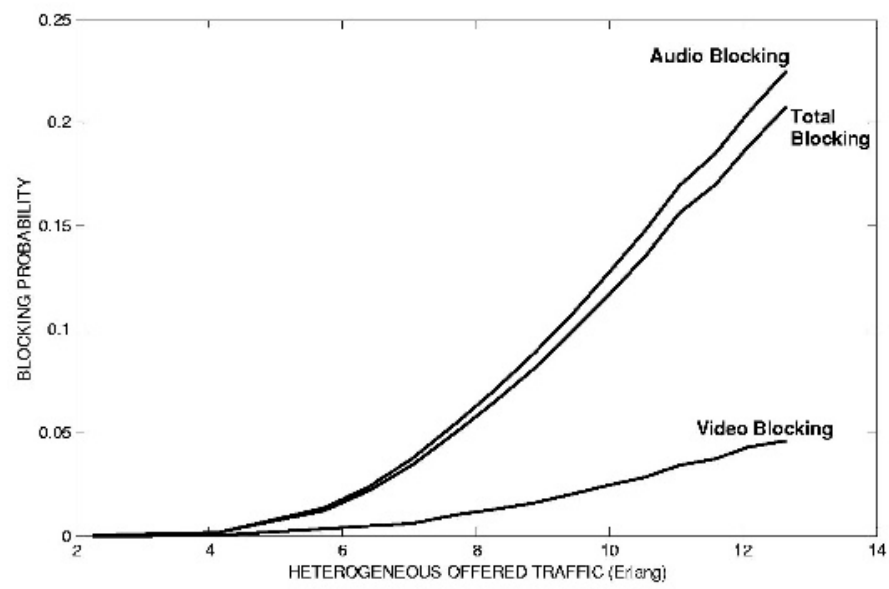

Fig. 1. Average audio, video and overall blocking probabilities for uniform FCA strategy versus heterogeneous offered traffic (Erlangs)

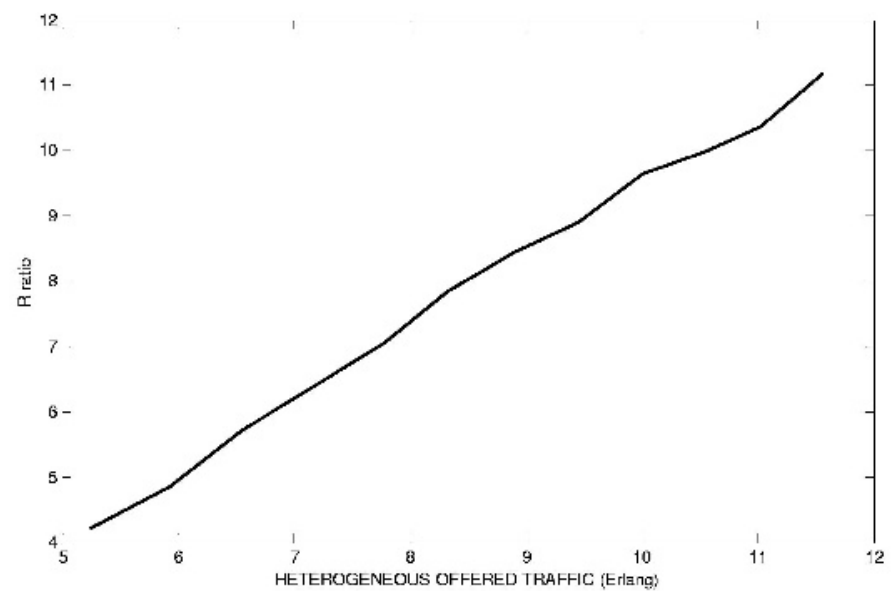

Fig. 2. Ratio of video call generation probability to audio call generation probability (R) for different values of average heterogeneous offered traffic (Erlangs) in the case of applying uniform FCA strategy

maximizing audio channel utilization while guaranteeing QoS requirement for video traffic and in general case this is accomplished using stochastic optimization techniques [9]. The main problem with this procedure is its mathematical complexity, which in some cases turns out to be completely untractable. In this research by adopting an approach similar to one outlined in [10], the analytical complexity of solution procedure has been considerably reduced.

Suppose new call arrivals for both audio and video traffic follow Poisson processes with mean arrival rates $\lambda_{\mathrm{A}}$ and $\lambda_{\mathrm{V}}$ and call servicing processes are also Poisson with mean servicing rates $\mu_{\mathrm{A}}$ and $\mu_{\mathrm{v}}$, respectively. 
If in the time origin $(t=0) n_{0}$ video channels out of total $N_{v}$ nominal video channels assigned to an arbitrary call are busy carrying video calls, then the probability that there will be $\mathrm{n}$ active video channels due to video traffic after time $\mathrm{t}$ in this cell is a transient state probability of a $\mathrm{M} / \mathrm{M} / \mathrm{N}_{\mathrm{v}} / \mathrm{N}_{\mathrm{v}}$ queuing system [11]. If the offered traffic load to the cell is not too heavy, this probability can be approximated by the transient state probability of a $M / M / \infty / \infty$ queuing system, $Q_{n}\left(t, n_{0}\right)$, according to the following equation: [10]

$$
P_{n}\left(t, n_{0}\right)=\frac{Q_{n}\left(t, n_{0}\right)}{\sum_{i=0}^{N_{V}} Q_{i}\left(t, n_{0}\right)}
$$

It can be shown that: $[10,11]$

$$
Q_{n}\left(t, n_{0}\right)=\left\{\begin{array}{l}
\frac{1}{n !} e^{\left(\beta-\mu_{v} n t\right)} \gamma^{n_{0}} \sum_{i=0}^{\infty} \frac{\left(n_{0}+i\right) !}{i !\left(n_{0}-n+i\right) !}(-\beta)^{i} \gamma^{i-n}, n \leq n_{0} \\
\frac{1}{n !} e^{\left(\beta-\mu_{v} n t\right)} \sum_{i=0}^{\infty} \frac{(n+i) !}{i !\left(n-n_{0}+i\right) !}(-\beta)^{n-n_{0}+i} \gamma^{i}, n>n_{0}
\end{array}\right.
$$

Where

$$
\begin{aligned}
& \beta=\left[\left(\lambda_{V} / \mu_{V}\right)\left(1-e^{\mu_{V} t}\right)\right] \\
& \gamma=\left(1-e^{-\mu_{V} t}\right)
\end{aligned}
$$

If the goal is to cross-borrow one video channel without violating QoS requirement of video traffic in the original cell, then the maximum time that one video channel can be cross-borrowed, $\mathrm{T}_{0}$, will be given by solving:

$$
P_{N_{V}-1}\left(T_{0}, n_{0}\right)=P_{B}
$$

Since call holding time for audio calls follows an exponential distribution, the probability that none of the active audio calls in the original cells will be completed within $\mathrm{T}_{0}$ is:

$$
P_{A}=\operatorname{Exp}\left[-\left(N_{A}+1\right) \mu_{A} T_{0}\right]
$$

By defining a reasonable threshold for $\mathrm{P}_{\mathrm{A}}$ as $\mathrm{P}_{\mathrm{T}}$, the request for cross-borrowing will be granted only if:

$$
P_{A} \leq P_{T}
$$

As the bandwidth required for carrying a video call is multiple times larger than bandwidth necessary for an audio call, we can further extend the cross-borrowing concept by permitting one video channel to be simultaneously cross-borrowed by multiple audio calls, if QoS criterion for video traffic servicing is satisfied. Suppose a video channel is cross-borrowed by an audio call request arrived at time origin $\mathrm{t}_{1}$ for duration of $\mathrm{T}_{0}$ (derived from Eq. 4) and second audio call requests arrives at time $\mathrm{t}_{2}$ before completion of the first audio call carried by the once cross-borrowed video channel. Keeping in mind that the call holding duration is exponentially distributed 
and therefore is without memory, the probability that time duration $T_{0}+t_{1}-t_{2}$ is sufficient for both audio calls to be completed can be considered as a criterion for granting second cross-borrowing request. In other words, to maintain QoS requirement of video traffic, the second cross-borrowing will be allowed, if the probability of at least one audio call is not finished during $T_{0}+t_{1}-t_{2}$ is equal to or less than $\mathrm{P}_{\mathrm{T}}$. This statement can be expressed as:

$$
\begin{aligned}
& e^{-\mu_{A}\left(T_{0}+t_{1}-t_{2}\right)\left(N_{A}+2\right)}+e^{-\mu_{A}\left(T_{0}+t_{1}-t_{2}\right)\left(N_{A}+1\right)} \\
& \cdot\left(1-e^{-\mu_{A}\left(T_{0}+t_{1}-t_{2}\right)\left(N_{A}+2\right)}\right) \leq P_{T}
\end{aligned}
$$

The afore-said criterion can be generalized for simultaneous cross-borrowing of one video channel by $n$ audio calls in a straightforward manner.

\section{Teletraffic Performance Evaluation of HCBA-UCB}

To simulate the performance of HCBA-UCB as realistically as possible, a novel discrete-event cellular network simulator was developed, which uses a wraparound topology. Simulation environment consists of 49 hexagonal cells arranged in a $7 * 7$ array. In initial assignment phase 10 audio channels and 5 video channels were preassigned to each cell according to a 7-cell reuse pattern uniform FCA strategy. In other words, total allocated bandwidth to the cellular system consists of 70 audio and 35 video channels. In the presented research it has been assumed that bandwidth of a video channel is 5 times larger than bandwidth of an audio channel. Servicing processes of audio and video calls are Poisson with mean service rates of 1/98 and $1 / 189$ calls per second, respectively.

The mechanism for advancing simulation time and guaranteeing that all events occur in correct chronological order is based on the Future Event List (FEL). FEL contains all event notices for events that have been scheduled to occur at a future time. Scheduling a future event means that at the instant an activity begins, its duration is somehow derived (usually according to a given pdf) and the end-activity event, together with its event time, is placed on the FEL. After the system snapshot at simulation time $\mathrm{t}_{1}$ has been updated, the simulation clock is advanced to simulation time $t_{2}$ and the event associated with this moment in the FEL will be executed. At the time $t_{2}$, new future events may or may not be generated (randomly, with probabilities $\mathrm{p}_{\mathrm{i}}$ and $1-\mathrm{p}_{\mathrm{i}}$ ), but if any are, they are scheduled by creating event notices and putting them in their proper position on the FEL. This process repeats until the simulation is over.

Fig. 1 depicts overall, audio and video call blocking probabilities versus average heterogeneous offered traffic (Erlangs). In extracting these results it has been assumed that the probability of arrival of an audio call is 9 times greater than the probability of arrival of a video call. Also channel assignment strategy is solely based on uniform FCA. As our final goal from using HCBA-UCB is to improve audio teletraffic performance of the system without imposing additional costs upon video QoS performance, by changing the ratio of video call generation probability to audio call generation probability $(\mathrm{R})$ for different amounts of average heterogeneous offered 


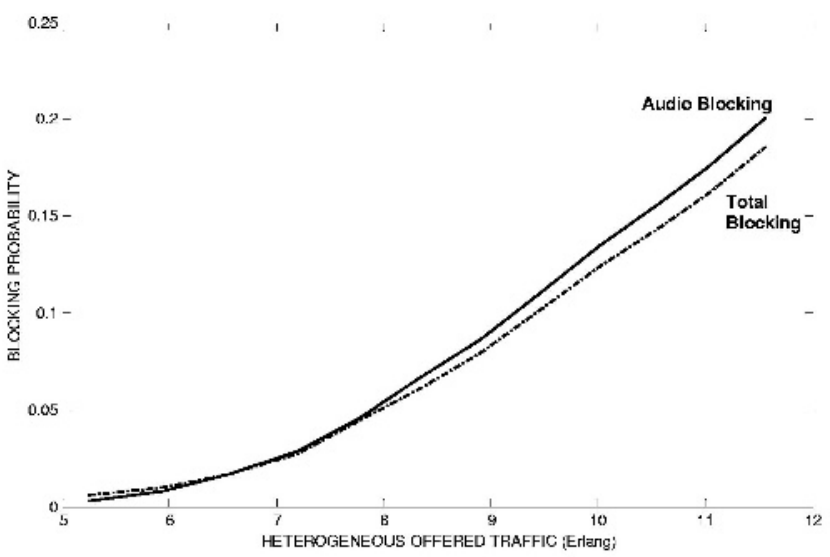

(a)

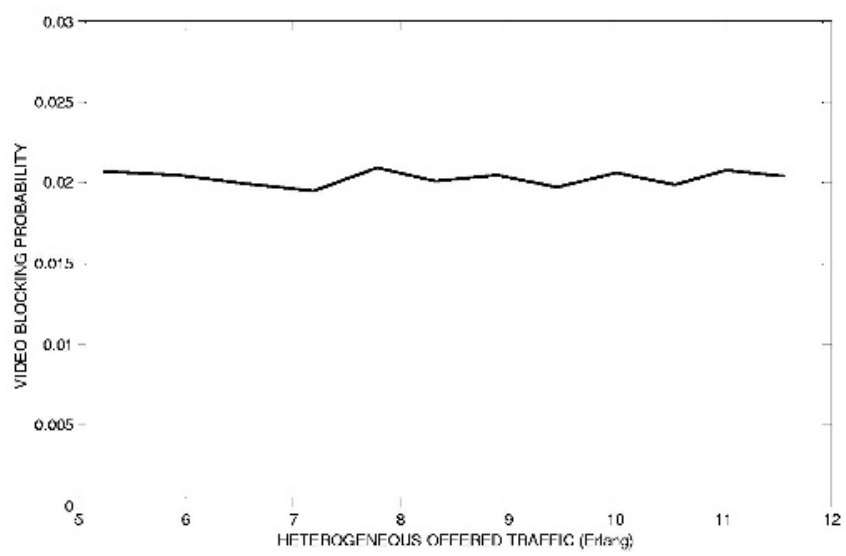

(b)

Fig. 3. (a) Audio, overall and (b) video average blocking probabilities for uniform FCA strategy corresponding to different values of $\mathrm{R}$ extracted from Fig. 2, versus heterogeneous offered traffic (Erlangs)

traffic in the case of uniform FCA strategy, the video call blocking probability has been kept fixed around $2 \%$ (with a tolerance of 0.002 ) while average heterogeneous offered traffic is increased. Fig. 2 shows different values of $\mathrm{R}$ obtained from this set of simulations and the corresponding overall, audio and video call blocking probabilities can be seen in Fig. 3 . 


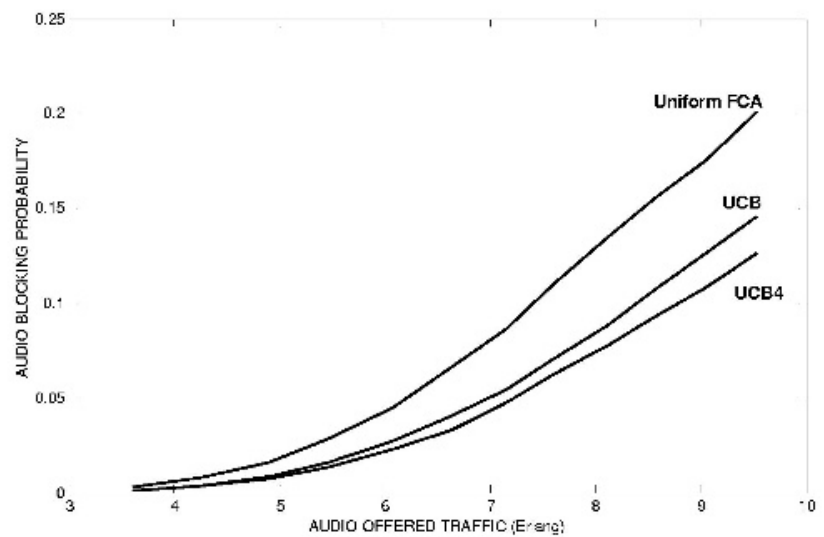

(a)

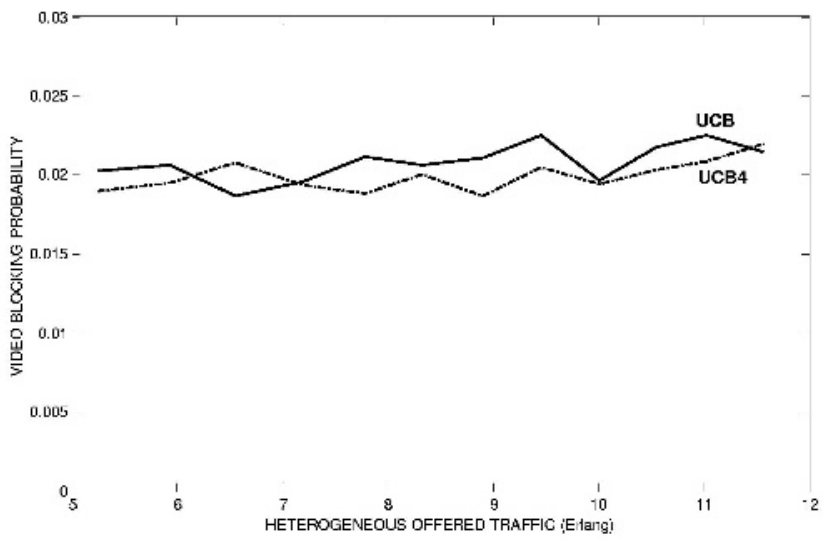

(b)

Fig. 4. (a) Audio average blocking probability versus audio offered traffic (Erlang) and (b) video average blocking probability versus heterogeneous offered traffic (Erlangs) for uniform FCA, UCB and UCB4 schemes, corresponding to different values of R extracted from Fig. 2

For assessing the mere effect of cross-borrowing on teletraffic performance of the cellular system, we applied this procedure to the uniform FCA strategy and the simulation results have been gathered in Fig. 4. By perceiving these results one can easily deduce that cross-borrowing concept has been successful in considerably improving audio teletraffic performance of the system without degrading video QoS performance. This deduction is even more perceptible when one cross-borrowed 


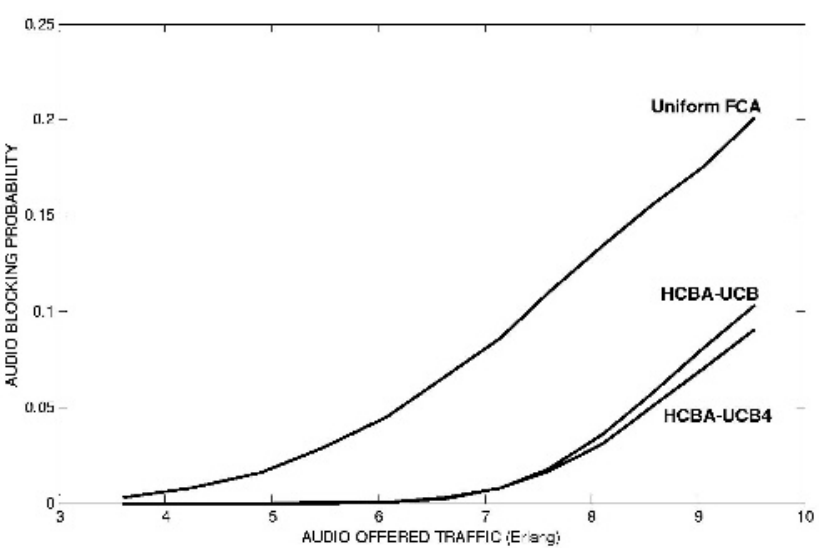

(a)

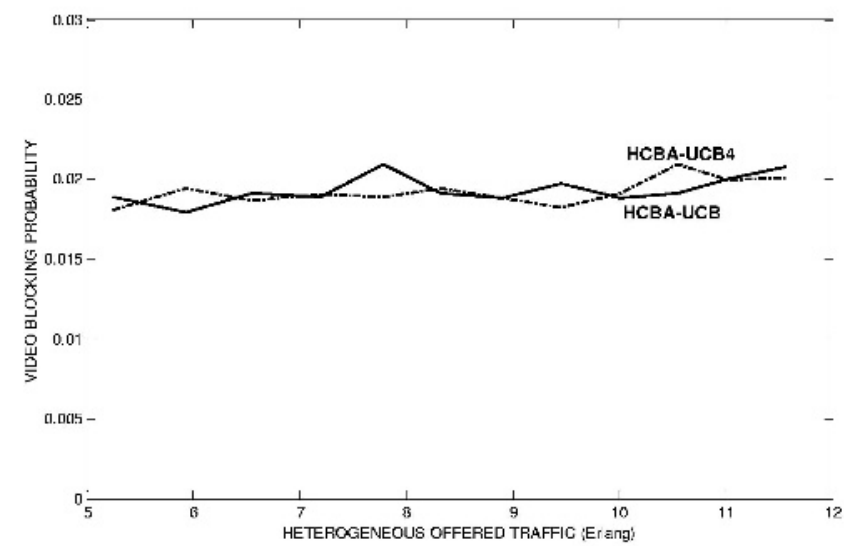

(b)

Fig. 5. (a) Audio average blocking probability versus audio offered traffic (Erlang) and (b) video average blocking probability versus heterogeneous offered traffic (Erlangs) for uniform FCA, HCBA-UCB and HCBA-UCB4 schemes,corresponding to different values of R from Fig. 2

video resource is allowed to simultaneously carry up to 4 audio channels (UCB4). In blocking index of 2\%, uniform FCA, UCB and UCB4 schemes can carry average offered audio traffics of 5.067, 5.65 and 5.95 Erlangs respectively, which can be interpreted as $12 \%$ and $17 \%$ increase in audio traffic carrying capacity for UCB and UCB4 over uniform FCA.

Finally, HCBA-UCB and HCBA-UCB4 (simultaneous cross-borrowing of a video resource by up to 4 audio call requests) schemes were subjected to a quantitative teletraffic performance evaluation. Fig. 5 depicts the obtained results in this regard. 
As it was expected, combination of traditional resource borrowing and crossborrowing concepts had an outstanding impact on audio teletraffic performance of the cellular system under simulation while negligibly affected its video teletraffic performance. In blocking index of 2\%, HCBA-UCB and HCBA-UCB4 can carry 7.57 and 7.8 Erlangs of average offered audio traffic, which respectively represent $49 \%$ and $54 \%$ increase in audio traffic handling capacity comparing to uniform FCA.

\section{Conclusion}

In this paper we proposed HCBA-UCB as a resource allocation scheme specifically developed for cellular environments with heterogeneous offered traffic. In a cellular network with heterogeneous offered traffic consisting of audio and video calls, HCBA-UCB uses intra-cell borrowing and inter-cell unilateral cross-borrowing to enhance teletraffic performance of the system. Cross-borrowing of a video resource by one or multiple audio calls will be allowed only if QoS requirement for video calls is not violated. Performance evaluation of HCBA-UCB indicates that this allocation scheme is capable of improving audio teletraffic performance of the cellular network while insignificantly affecting video QoS performance.

\section{References}

1. J.Korhoner, Introduction to 3G Mobile Communications, MA, Artech House, 2001

2. B.Li et.al., "On the performance of channel assignment strategies in multi-service wireless cellular networks", 5th Int. Symp. on Wireless Personal Multimedia Comm., 2002, pp. 1379-1384

3. L.Jorguseski et al, "Radio resource allocation in third generation mobile communication systems", IEEE Communication Magazine, Feb. 2001, pp. 117-123

4. S.Dixity et al, "Resource management and quality of service in third generation wireless networks", IEEE Communication Magazine, Feb. 2001, pp. 125-133

5. M.Casoni et al, "Admission control in T/CDMA systems supporting voice and data applications", IEEE Trans. on Wireless Comm., Vol. 1, No. 3, July 2002, pp. 540-548

6. M.El-Kadi, S.Olariu \& H.Abdel-Wahab, "A rate-based borrowing scheme for QoS provisioning in multimedia wireless networks", IEEE Trans. on Parallel \& Distributed Sys., Vol. 13, No. 2, Feb. 2002, pp. 156-166

7. L.Ortigoza-Guerrero \& A.H.Aghvami, Resource Allocation in Hierarchical Cellular Systems, MA, Artech House, 2000

8. M.Zhang \& T.P.Yum, "The non-uniform compact pattern allocation algorithm for cellular mobile systems", IEEE Trans. on Veh. Technol., Vol. 40, May 1991, pp. 387-391

9. J.Choi \& J.A.Silvester, "A fair-optimal channel borrowing scheme in multi-service cellular networks with reuse partitioning", IEEE Int. Conf. on Universal Personal Comm. (ICUPC'98), 1998, pp. 261-265

10. T.S.P.Yum \& M.Chen, "Dynamic channel assignment in integrated-services cable networks", IEEE Trans. on Comm., Vol. 42, No. 2/3/4, 1994

11. D.Gross \& C.M.Harris, Fundamentals of Queueing Theory, 2nd Ed., John Wiley \& Sons, 1985 